\title{
MACH-ZEHNDER INTERFEROMETER INVESTIGATIONS ON SOME LASER DYE SOLVENTS
}

\begin{abstract}
R. GHAZY
Laser Lab., Phys. Dept., Faculty of Sciences, Tanta University, Tanta, Egypt

(Received April 8, 1998; revised version October 16, 1998;

in final form February 18, 1999)

We have used a laser interferometric method to determine the refractive index of some laser dye solvents with a high accuracy of $5.8 \times 10^{-4}$. The determination of refractive index $(n)$ has been done by counting the interference fringes $(N)$ as a function of the incidence angle of the laser beam. The absolute refractive index of pure water, dimethylsulfoxide, and different mixtures of percentages $(P) 25 \%, 50 \%, 75 \%$ of dimethylsulfoxide has been measured. The relation between the bulk refractive index $\left(n_{\mathrm{m}}\right)$ of these mixtures is plotted as a function of the investigated percentages. The temperature behavior of these refractive indices $\left(\mathrm{d} n_{\mathrm{m}} / \mathrm{d} t\right)$ was studied carefully within a temperature range of $\left(20-80^{\circ} \mathrm{C}\right)$. We have found that both the refractive index $\left(n_{\mathrm{m}}\right)$ and temperature behavior of the refractive indices $\left(\mathrm{d} n_{\mathrm{m}} / \mathrm{d} t\right)$ of different mixtures of the investigated solvents have the average index properties of the parent solvents. Also, a fitted empirical relation between $n_{\mathrm{m}}, \mathrm{P}$, and $t$ has been assumed as $n_{\mathrm{m}}=a P^{b} t^{c}$ with empirical constants $a, b$, and $c$. According to that equation we have recalculated $n_{\mathrm{m}}$ of these solvent mixtures and we have found satisfying agreement with the experimental results.
\end{abstract}

PACS numbers: $42.55 . \mathrm{Mv}, 78.20 . \mathrm{Ci}$

\section{Introduction}

When the pump laser beam is incident upon the dye solution it is absorbed causing a heat change of the solution. The density of the solution will change leading to a change of the refractive index $n$ of the solution. The formation of the refractive-index gradient due to non-uniform heating by the pump radiation in the region of the optical gain gives a major limitation on achieving high power, spectrally narrow and spatially coherent radiation from the lasers. One of the main mechanisms by which the pump radiation is converted into heat in the dye solution 
is radiationless deactivation of molecules in excited states, the proportion of which determines the dyes fluorescence efficiency [1].

Also, the environmental temperature causes the same effects like those caused by the pump radiation conversion into heat in the solution. The inhomogeneous heating causes an inhomogeneous distribution of the refractive index of the dye solution which in turn causes a thermal lensing effect leading to the instability of the optical system and a reduction of the maximum pump power. In addition, the temperature change of the laser dye will defocus the laser beam when $\mathrm{d} n / \mathrm{d} t<0$ or focus the beam when $\mathrm{d} n / \mathrm{d} t>0$. The rate of change of the refractive index of the solvent with temperature $\mathrm{d} n / \mathrm{d} t$ is a most useful parameter to achieve the thermo-optical properties of a solvent. The accurate value of $|\mathrm{d} n / \mathrm{d} t|$ is essential to determine the dependence of the critical pump intensity $P_{\text {crit }}[1,2]$ which is given as

$$
P_{\text {crit }} \leq(\sqrt{2 \pi} \rho c) /\{4 \delta[1-\exp (-y l)]|\mathrm{d} n / \mathrm{d} t|\} \exp \left(2 y^{2} / \omega_{A}^{2}\right)\left(v \omega_{A}^{3} / S\right),
$$

where $S$ is the stability range of the unperturbed dye laser resonator, $\delta$ is the percentage value of the pump power which is converted into heat, $\rho$ - density, $l$ - the thickness of the jet stream, $v$ - the flow velocity, and $\omega_{A}$ - the beam radius of the focused pump laser.

The values of both $n$ and $\mathrm{d} n / \mathrm{d} t$ of the mixtures are studied carefully by using a Mach-Zehnder interferometer (MZI) with a high degree of accuracy and are reported in the present paper.

\section{Experimental method}

The Mach-Zehnder interferometer is an analogue to the Michelson interferometer (MI) which is based on the two-beam interference by amplitude splitting of the incoming wave. The construction of MZI is shown in Fig. 1. The two waves travel along different paths.

When a transparent object is inserted into one arm of the interferometer, it alters the optical path difference between the two beams. This causes a change of the interference pattern, which enables us to determine accurately the refractive index of the sample under investigation and the local variation of the interfering fringes. Therefore the MZI may be regarded as a sensitive refractometer.

When the beam splitters $B_{1}, B_{2}$ and the mirrors $M_{1}, M_{2}$ are all completely parallel, the path difference between the two split beams does not depend on the angle of incidence $\phi$, because the path difference $\delta_{1}=B_{1} M_{1}=2 a \cos \phi$ because the beams 1 and 3 are exactly compensated by the sample path length between $M_{2}$ and $B_{2}$ (Fig. 1b). This means that the interfering waves in the symmetric interferometer (without sample) experience the same path difference on the solid path as on the dashed path in Fig. 1a. Without the sample the total path difference is therefore zero, it is $\Delta s=(n-1) L, L$ is the sample length, with the sample having the refractive index $n$ in one arm of the interferometer [3].

The Mach-Zehnder interferometer has been used as an accurate measurement tool to determine the refractive index $n$ of the solvents [4]. The refractive index of both pure water and the dimethylsulfoxide (DMSO) has been determined at the ambient temperature and then as a function of temperature. The solvent 

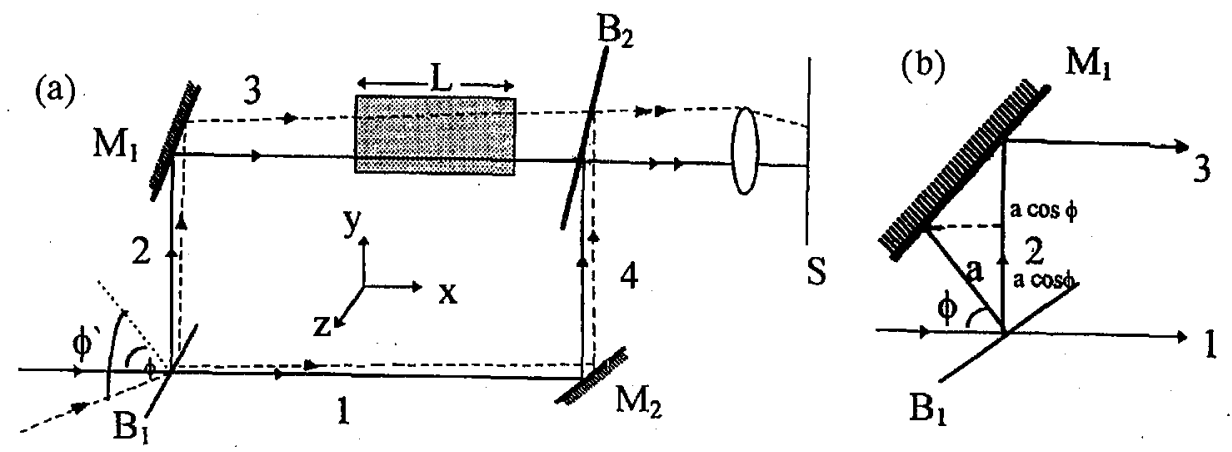

Fig. 1. Optical layout of Mach-Zehnder interferometer (a). Path difference between two parallel beams in MZI (b).

mixtures have been done from DMSO and pure water with different percentages $P$. The refractive index of those different mixtures has been measured at different temperatures. Each sample investigated was contained within a rectangular quartz cell located in one of MZI's arms. The light source of the interferometer is the $\mathrm{He}-\mathrm{Ne}$ laser with a $632.8 \mathrm{~nm}$ wavelength. The cell was fixed on the base plate of a spectrometer to enable us to change the incidence angle of laser beam with a small fraction of degree down to 10 minutes. The whole range of the incidence angle was 5-30 degrees with a step of 30 minutes measured at both right and left sides of the beam to reduce the error as possible. The solvent refractive index has been determined with a high degree of accuracy $5.8 \times 10^{-4}$. By counting the number of the interference fringes $N$ produced as a function of the incidence angle $\phi$ of the expanded laser beam, one can determine the refractive index $n$ of the given sample by the graphical representation [5] of the following relation:

$$
n=\left[(t-N \lambda)(1-\cos \phi)+\left(N^{2} \lambda^{2} / 2 t\right)\right] /[t(1-\cos \phi)-N \lambda],
$$

where $\phi$ is the angle of incidence, $N$ - the number of fringes, $t$ - the cell thickness, $10 \mathrm{~mm}, \lambda$ - the wavelength of the laser source, $632.8 \mathrm{~nm}$. The term $N^{2} \lambda^{2} / 2 t$ can be neglected [6] as it is very small.

The experimental arrangement of refractive index determination is shown in Fig. 2. Each of the two mirrors $M_{1}$ and $M_{2}$ is fixed on three-dimensional holder (movable in three dimensions by micrometer screws) to enable us to get a fine adjustment. The whole system is built on a rigid garnet plate with $42 \mathrm{~mm}$ in thickness which is mounted on an invader plate with a thickness of $79 \mathrm{~mm}$. The invar plate is fixed on four thick rubber rings. This isolation enables us to get a vibrationless interference fringes leading to a high accuracy determination of the refractive index.

In order to determine the value of $\mathrm{d} n / \mathrm{d} t$ for a solvent the change of the number of fringes has been measured as the dye solvent temperature was changing by using a thermal heating unit. Equation (1) was used to deduce the associated change in refractive index $\Delta n$. At normal incidence of the laser beam $(\phi=0)$ the neglected term of Eq. (2) can be used at a temperature $T_{1}$ and then at a temperature $T_{2}$. Then the change in the number of fringes $\Delta N$ which cross the field of view corresponding to the change of sample temperature $\Delta t$ can be counted. 


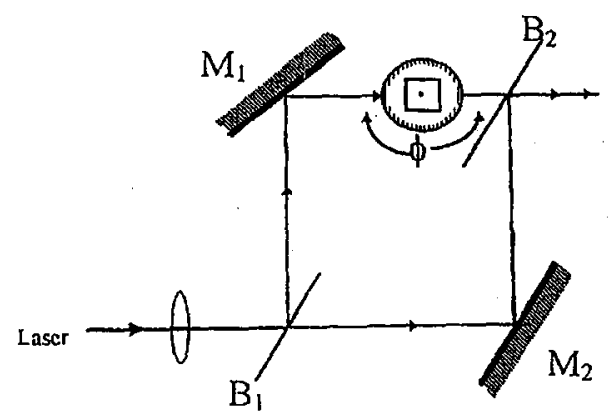

Fig. 2. Optical arrangement of MZI for determination of refractive index.

Consequently, the change of the refractive index $\Delta n$ is determined as a function of the change in the number of fringes $\Delta N$ as follows:

$$
\Delta n=[(\Delta N) / 2 t] \lambda \text {. }
$$

The thermal heating unit consists of two concentric stainless steel cylindrical cells, its design is included in Fig. 3 . Figure 3 shows the experimental arrangement for the determination of heat change of refractive index $\mathrm{d} n / \mathrm{d} t$. The sample under investigation is contained inside the central cell between two parallel glass transparent windows. The outer cylinder cell is used to heat the circulated water which heats the sample in turn. The heated water comes from a copper reservoir which is heated by an electrical unit with a gradually increasing rate by using a special power supply. The temperature is calibrated and controlled through the power

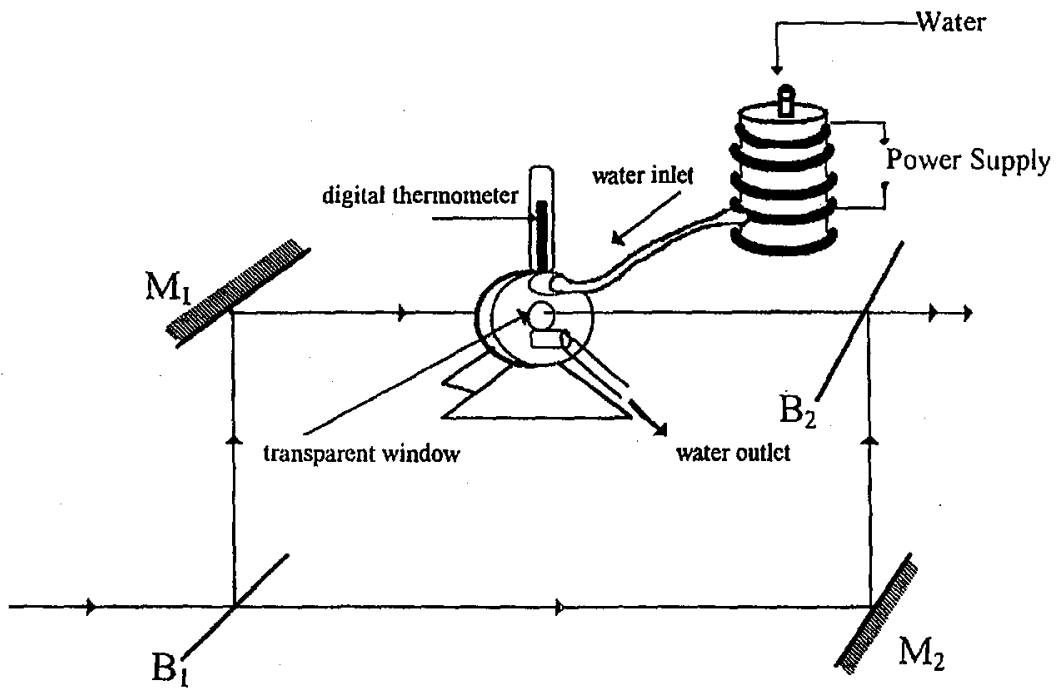

Fig. 3. Experimental arrangement for studying the dependence of the refractive index on the temperature $\mathrm{d} n / \mathrm{d} t$. 
supply adjustment. The temperature is measured by a digital thermometer and also by a special thermocouple with an accuracy of $\pm 0.5^{\circ} \mathrm{C}$.

Then by calculating the refractive index $n$ which corresponds to each value of temperatures one can plot the relationslip between $n$ and $T$ from which $\mathrm{d} n / \mathrm{d} t$ is calculated.

The number of fringes is counted manually by the visualization method with an accuracy $5.8 \times 10^{-4}$. The accuracy is determined as the mean average of the two following terms:

1. The angle term:

accuracy $=$ the smallest angle could be measured/the whole angle range measured $=1$ minute $/ 30$ degree $=5.5 \times 10^{-4}$.

2. The fringes term:

accuracy $=$ the smallest part of the fringe could be measured/the largest number of fringes counted $=0.5$ (mid-point between two lighting successive fringes) $/ 820=6.1 \times 10^{-4}$.

Therefore, the total accuracy becomes $5.8 \times 10^{-4}$.

\section{Theoretical background}

\subsection{Mean polarizability and dielectric constant}

The mean polarizability $\alpha$ can be determined as a function of refractive index $n$ of a sample in accordance to the following relation:

$$
\alpha=(3 / 4 \pi M)\left[\left(n^{2}-1\right) /\left(n^{2}+2\right)\right],
$$

where $M$ is the number of molecules per unit volume.

By using the Maxwell relation between the dielectric constant $\varepsilon$ and the refractive index $n$ for a non-polar material it can be shown that

$$
\varepsilon=n^{2} \text {. }
$$

Therefore, Eq. (4) can be rewritten as

$$
\alpha=(3 / 4 \pi M)[(\varepsilon-1) /(\varepsilon+2)],
$$

i.e. the mean polarizability $\alpha$ can be determined as a function of either $n$ and $M$ or $\varepsilon$ and $M$.

For a polar material the following equation can be used to calculate the. dielectric constant $\varepsilon$ and the permanent dipole moment $\mu_{0}$ of the molecule under investigation [7]

$$
\begin{aligned}
& {[(\varepsilon-1) /(\varepsilon+2)]-\left[\left(n^{2}-1\right) /\left(n^{2}+2\right)\right]=\left[3 \varepsilon\left(n^{2}+2\right) /\left(2 \varepsilon+n^{2}\right)(\varepsilon+2)\right]} \\
& \times\left(4 \pi M \mu_{0}^{2} / 9 k T\right)=\left(4 \pi M \mu_{0}^{2} / 9 k T\right) f\left(\varepsilon, n^{2}\right)
\end{aligned}
$$

with

$$
f\left(\varepsilon, n^{2}\right)=3 \varepsilon\left(n^{2}+2\right) /\left(2 \varepsilon+n^{2}\right)(\varepsilon+2),
$$

where $\mu_{0}$ is the permanent dipole moment of the molecule, and $k$ is the Boltzmann constant. 


\subsection{Molar refractivity $A$}

The molar refractivity $A$ is essentially the total polarizability of a mole of the substance [8], being defined as

$$
A=(4 \pi / 3) N_{\text {Avo }} \alpha,
$$

where $N_{\text {Avo }}$ is the Avogadro number.

For monoatomic substances it is called the atomic refractivity.

In terms of the molecular weight $W$, the density $\rho$, and the refractive index $n$ of the substance, the molar refractivity $A$ can be written as

$$
A=(W / \rho)\left[\left(n^{2}-1\right) /\left(n^{2}+2\right)\right] .
$$

The quantity

$$
r=\left[\left(n^{2}-1\right) /\left(n^{2}+2\right)\right](1 / \rho)
$$

is called the specific refraction of a substance. For a given substance, $r$ is independent of $\rho$. Therefore [9]

$$
A=r W \text {. }
$$

\subsection{Molar refractivity of a mixture}

The molar refractivity of a mixture of two substances is equal to the sum of the contributions due to each substance. Thus, if two liquids of refractivities $A_{1}$ and $A_{2}$ are mixed, and if a unit volume of the first liquid contains $N_{1}$ molecules and of the second $N_{2}$ molecules, then the molar refractivity of the mixture will be

$$
A=\left(N_{1} A_{1}+N_{2} A_{2}\right) /\left(N_{1}+N_{2}\right) \text {. }
$$

Finally, we consider the dependence of the molar refractivity $A$ of a compound on the atomic refractivities of its constituent atoms. If the molecule consists of $N_{1}$ atoms of refractivity $A_{1}$ and of $N_{2}$ atoms of refractivity $A_{2}$, etc., then obviously

$$
A=N_{1} A_{1}+N_{2} A_{2} .
$$

\section{4. $n_{\mathrm{m}}$ of a mixture}

For a mixture of two different liquids, the refractive index of that mixture $n_{\mathrm{m}}$ is given by $[10]$

$$
\begin{aligned}
& \left(n_{\mathrm{m}}{ }^{2}-1\right) /\left(n_{\mathrm{m}}{ }^{2}+2\right)=\left\{\left[\left(A_{1} / W_{1}\right)\right] f_{1}+\left[\left(A_{2} / W_{2}\right) f_{2}\right]\right\} \rho_{\mathrm{m}} \\
& =(A / W)_{\mathrm{m}} \rho_{\mathrm{m}},
\end{aligned}
$$

where $A_{1}, A_{2}$ are the molar refractivities of molecules of type 1 and $2 ; W_{1}, W_{2}$ are the molecular weights of molecules of type 1 and $2 ; f_{1}, f_{2}$ are the fractions, by mass, of molecules of type 1 and $2 ; n_{\mathrm{m}}$ is the refractive index of the mixture, and $\rho_{\mathrm{m}}$ is the density of the mixture.

\subsection{Temperature behavior $d n / d t$}

Both the density and the refractive index are functions of temperature $t$, but molar refractivity is expected to be independent of temperature [11]. So that we have 


$$
\left[n_{\mathrm{m}}(T)-1\right] /\left[n_{\mathrm{m}}^{2}(T)+2\right]=(A / W)_{\mathrm{m}} \rho_{\mathrm{m}}(T) .
$$

If we assume that the refractivity of a liquid is constant, $\mathrm{d} n / \mathrm{d} t$ may be estimated from the density, refractive index and $\mathrm{d} \rho / \mathrm{d} t$, from the above equation we can get

$$
\mathrm{d} n_{\mathrm{m}} / \mathrm{d} T=(A / W)_{\mathrm{m}}\left[\left(n^{2}{ }_{\mathrm{m}}+2\right) / 6 n_{\mathrm{m}}\right]\left(\mathrm{d} \rho_{\mathrm{m}} / \mathrm{d} T\right) .
$$

But

$$
(A / W)_{\mathrm{m}}=\left(1 / \rho_{\mathrm{m}}\right)\left[\left(n_{\mathrm{m}}{ }^{2}-1\right) /\left(n_{\mathrm{m}}^{2}+2\right)\right] .
$$

Therefore the macroscopic properties of both density and refractive index of the liquid can be used to determine $\mathrm{d} n_{\mathrm{m}} / \mathrm{d} t$ according to Eq. (16).

The dimethylsulfoxide solvent is one of the strong organic solvents for laser dyes. Both water and DMSO belong to the three main categories of solvents for dissolving organic dyes. These categories are $[1,2,11]$ :

1. Water based solvents, e.g., pure water or water based solutions such as dimethylsulfoxide;

2. Simple organic solvents, e.g., methanol, ethanol, ethylene glycol, DMSO, and a mixture of them; and

3. Mixture of water-based solvents and organic solvents, i.e. categories (1) and (2) together produce this third class of laser dye solvents.

The importance of this class of solvents lies in the ability of getting a high solubility of the laser dye and a suitable thermo-optical characteristics.

\section{Results and discussions}

\subsection{Water-based solvents}

Water-based laser dye solvents are considered as excellent solvents because of their thermo-optical characteristics. In comparison with other liquids they have a smaller value of $\mathrm{d} \rho / \mathrm{d} t$ and consequently a smaller value of $\mathrm{d} n / \mathrm{d} t$ as shown from Eq. (16). It is known that the water has a maximum density at about $4^{\circ} \mathrm{C}$ which leads to $\mathrm{d} \rho / \mathrm{d} t=0$. Therefore, if the temperature of the water about $4^{\circ} \mathrm{C}$ is changed with a small fraction one can expect that $\mathrm{d} n / \mathrm{d} t=0$, büt there is some doubt about the exact temperature at which this occurs [10]. This particular property of water is the reason beyond considering it as the best thermo-optical solvent. The method of determination of the refractive index $n$ of water is shown in Fig. 4. The obtained value of $n$ is shown in Table.

Figure 5 shows the dependence of the measured refractive index $n$ as a function of temperature for pure water. It was shown that around $4^{\circ} \mathrm{C}$ there is a very little change in refractive index with temperature [11]. This result agrees with another author [12]. We used our measured values of the refractive index $n$ of water and the tabulated values of CRC [13] for density $\rho$ at different temperatures to calculate the molar refractivity $A$ of water. The values of $A$ for water are plotted against the temperature in Fig. 6 . The molar refractivity of water is constant at a value of 3.71 which agrees very well with the other authors $[11,13,14]$. Because of the tendency of dye molecules to form dimers, the dye laser based on aqueous 


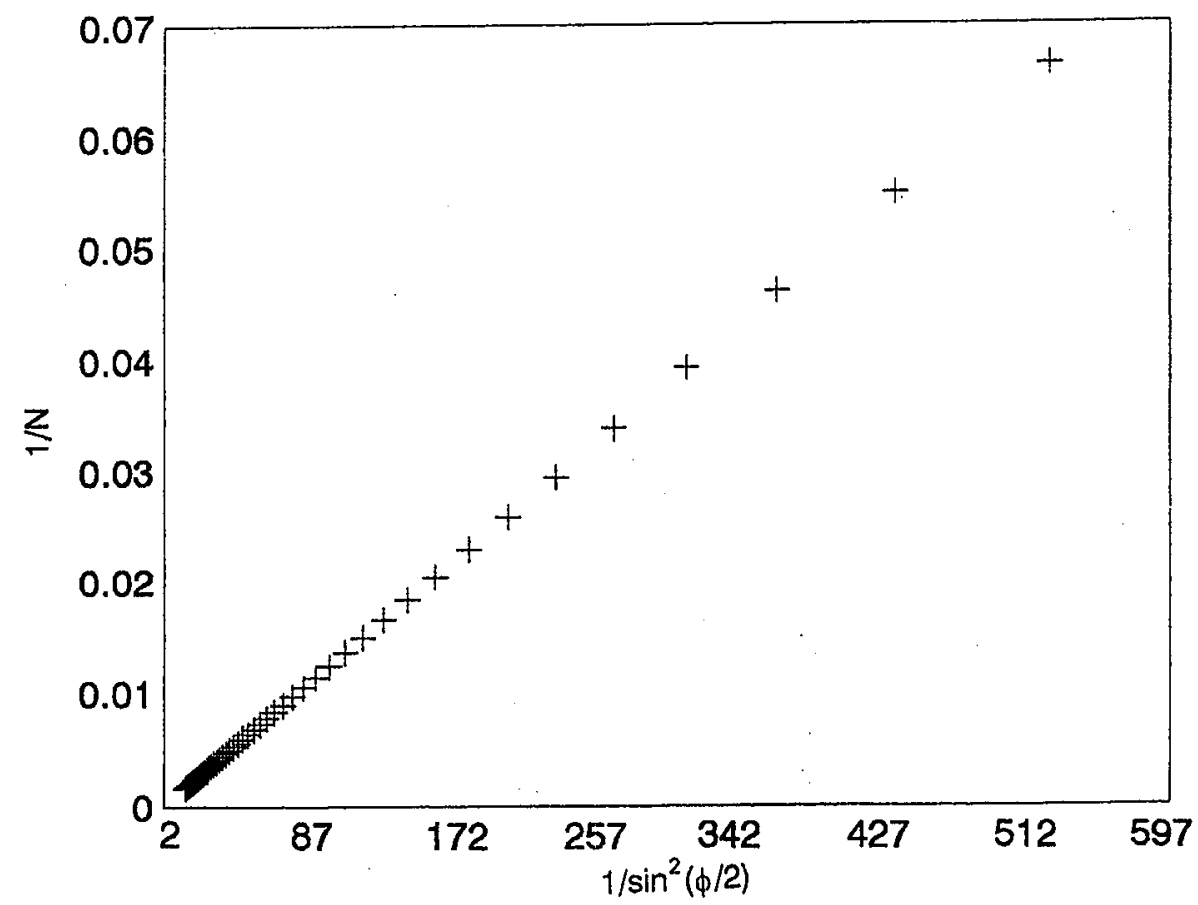

Fig. 4. The relation between number of fringes $N$ and the angle of incidence $\phi$ to determine the refractive index $n$ of pure water.

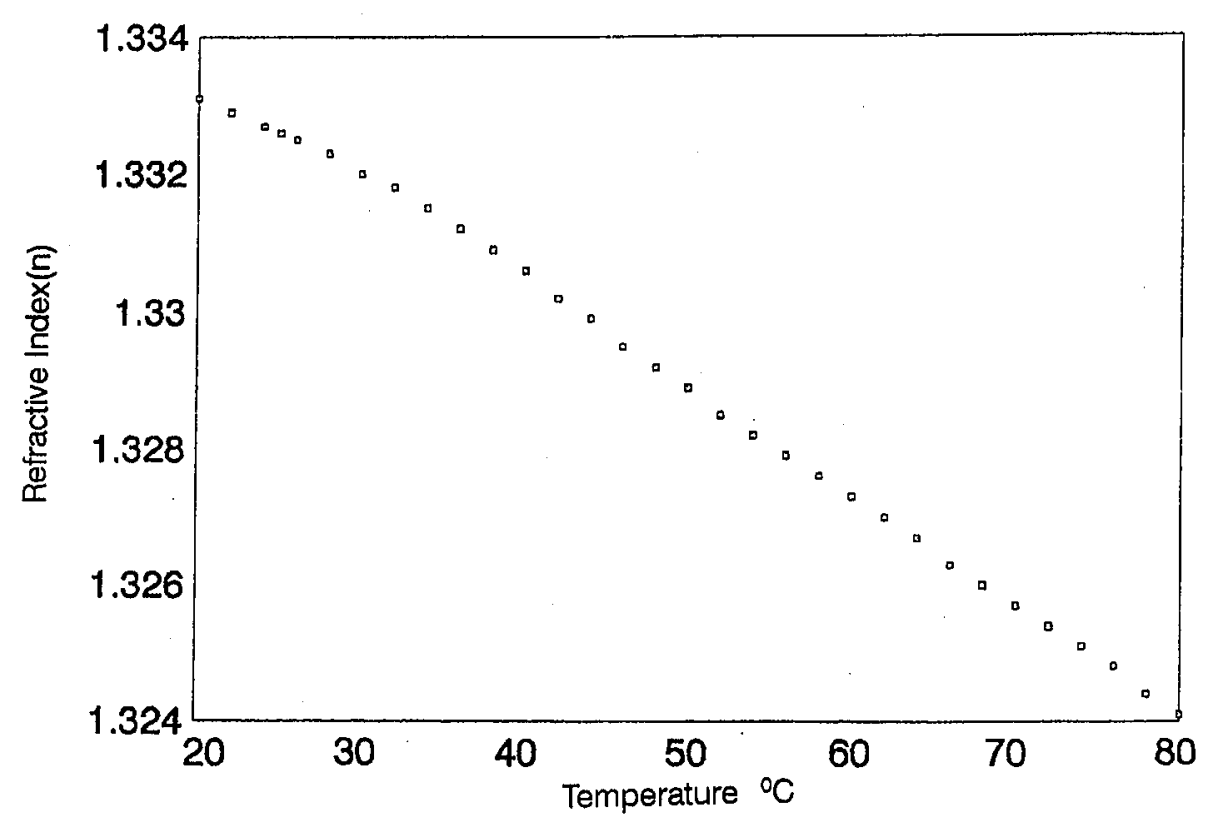

Fig. 5. Thermal behavior of the refractive index of pure water to determine $\mathrm{d} n / \mathrm{d} t$. 
TABLE

Comparison between the measured and theoretical values of $n$ and $\mathrm{d} n / \mathrm{d} t$.

\begin{tabular}{c|c|c|c|c}
\hline \hline$P(\%)$ & $n_{\exp }$ & $n_{\mathrm{th}}$ & $(\mathrm{d} n / \mathrm{d} t)_{\exp }$ & $(\mathrm{d} n / \mathrm{d} t)_{\mathrm{th}}$ \\
\hline 0 & 1.3326 & - & $1.55 \times 10^{-4}$ & - \\
25 & 1.3687 & 1.35939 & $1.28 \times 10^{-4}$ & $1.19 \times 10^{-4}$ \\
50 & 1.4047 & 1.40743 & $1.020 \times 10^{-4}$ & $1.033 \times 10^{-4}$ \\
75 & 1.4475 & 1.43632 & $1.395 \times 10^{-4}$ & $1.386 \times 10^{-4}$ \\
100 & 1.4768 & - & $1.19 \times 10^{-4}$ & -
\end{tabular}

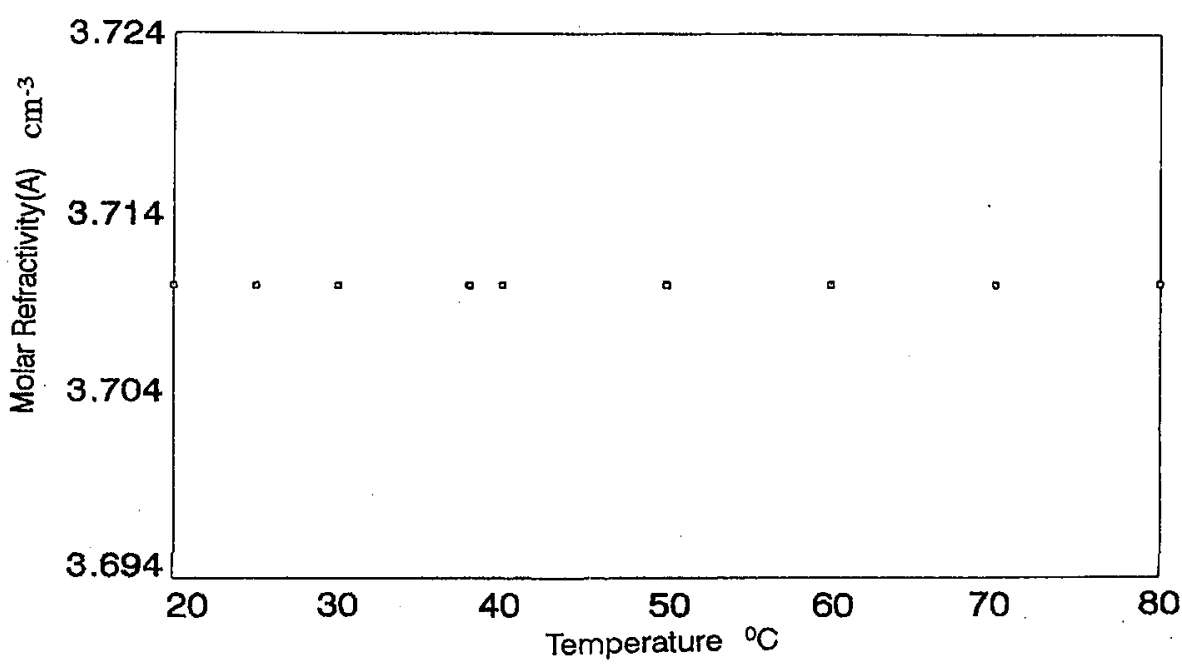

Fig. 6. The relationship between molar refractivity $A$ and temperature $T$ of water.

solutions has a low efficiency. The absorption spectra of these formed dimers lie in the absorption region of the lasing monomers and also frequently overlap their fluorescence band hence reducing the fluorescence efficiency. In the case of using organic solutions the dimerization is slight and the efficiency of the dye laser produced is much better. Therefore to reduce the dimerization process some organic compounds may be added to water.

\subsection{DMSO organic solvent}

The dimethylsulfoxide is one of the strongest organic solvents, which belongs to the class (2) of dye solvents. The determination of the refractive index of DMSO is shown in Fig. 7 with the values given in Table. The temperature behavior of the refractive index for this solvent within a temperature range of $\left(20-80^{\circ} \mathrm{C}\right)$ is shown in Fig. 8. The value of $\mathrm{d} n / \mathrm{d} t$ is given in Table. This kind of organic liquid does not have the same behavior like water since it has a normal density-temperature behavior, i.e., its density decreases linearly by expansion, with increasing temperature. 


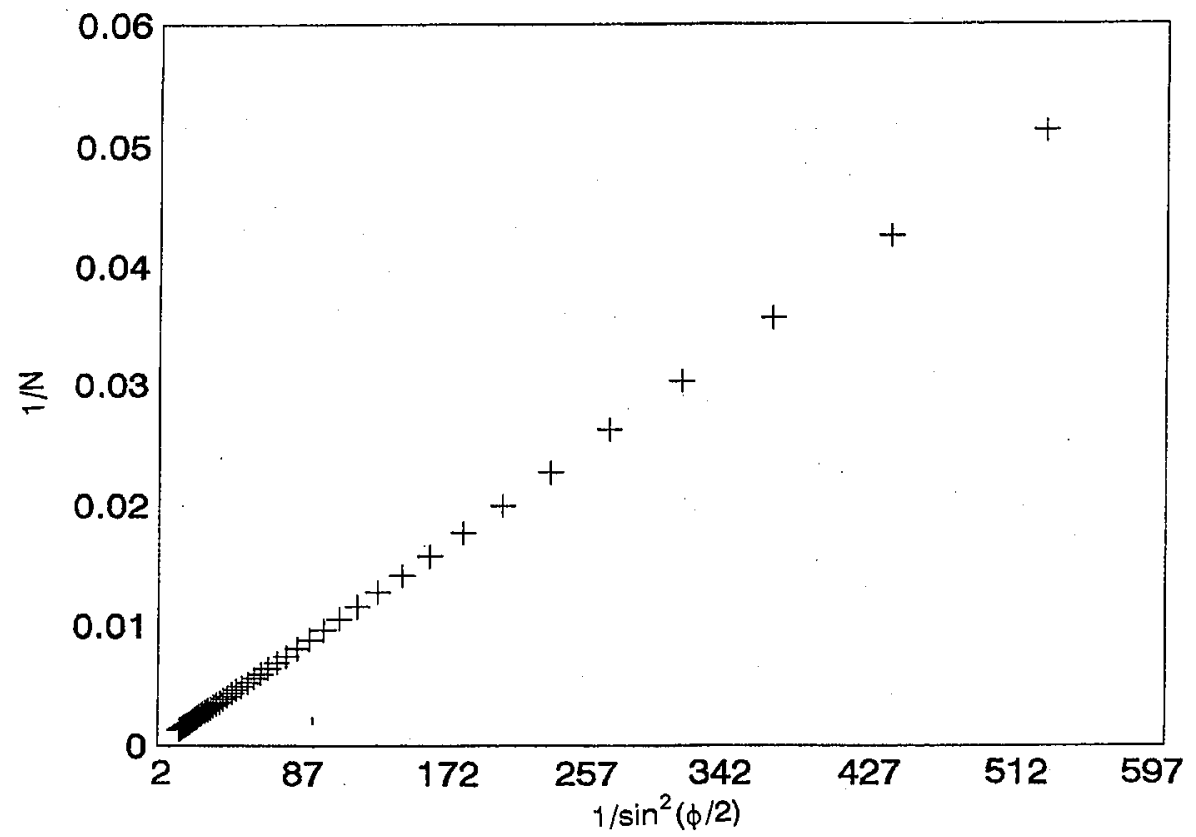

Fig. 7. Determination of the refractive index $n$ of dimethylsulfoxide by using MZI method.

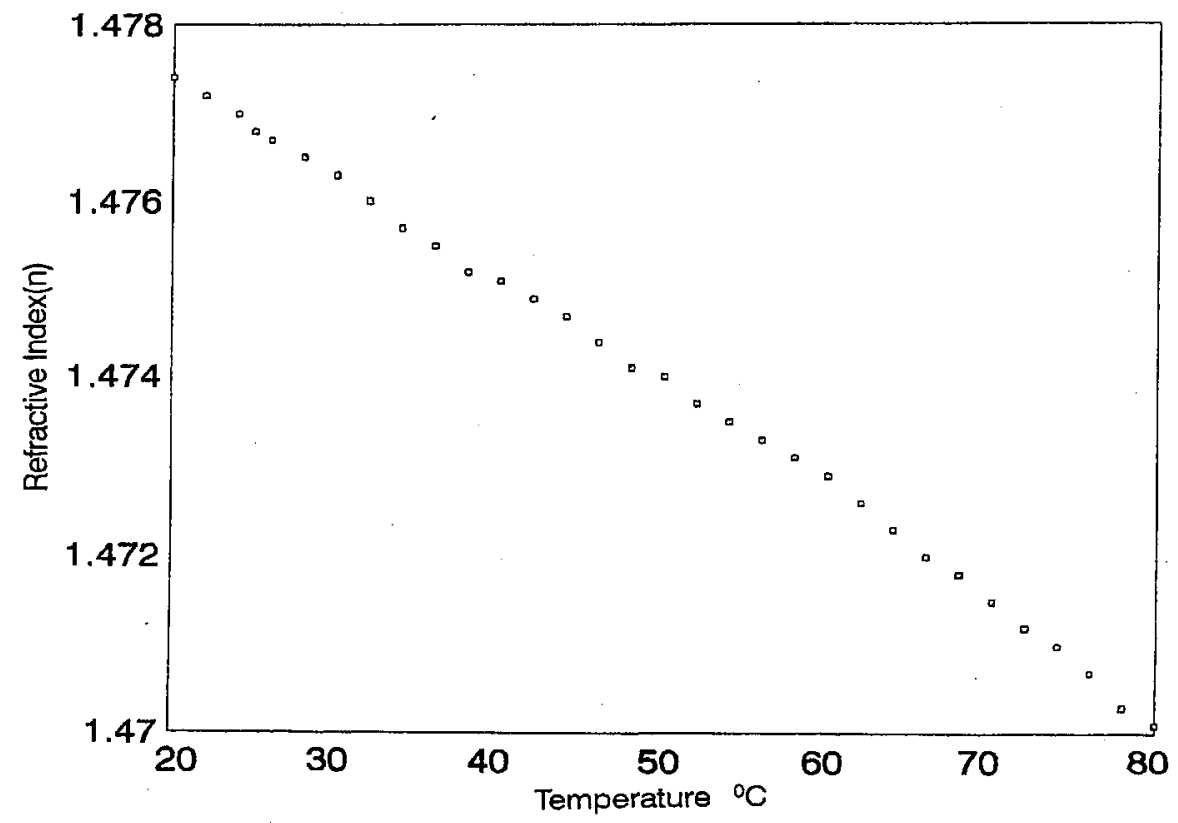

Fig. 8. Thermal behavior of the refractive index of dimethylsulfoxide. 


\subsection{Solvent mixtures}

The third class of laser dye solvents can include mixtures of water-based solvents, or mixtures of water-based and organic solvents. The investigated mixtures in the present work belong to this category. We added DMSO to water with different percentages $(0 \%, 25 \%, 50 \%, 75 \%, 100 \%$ DMSO). During the determination of the number of interference fringes of those mixtures $N_{\mathrm{m}}$ we have found that, at the same angle of laser beam incidence $\phi$,

$$
N_{\mathrm{m}}=N_{\mathrm{s} 1} P_{1}+N_{\mathrm{s} 2} P_{2},
$$

where $N_{\mathrm{s} 1}, N_{\mathrm{s} 2}$ are the numbers of the interference fringes of the solvents 1 and 2; $P_{1}, P_{2}$ are the percentages of the two mixed solvents.

The refractive index of each percentage is determined as shown in Fig. 9 and the relation between the percentages $P$ and the refractive index $n_{\mathrm{m}}$ is plotted in Fig. 10 from which it can be obviously shown that there is a direct proportionality between the refractive index $n_{\mathrm{m}}$ of the mixture and the percentage of the organic solvent (DMSO) which had been added to the water. The types of temperature behavior of the mixtures are plotted on one diagram shown in Fig. 11. In Figs. 10 and 11 we can see that the index properties $(n, \mathrm{~d} n / \mathrm{d} t)$ of a mixture is given by an average of the index properties of the parent solvents. Since

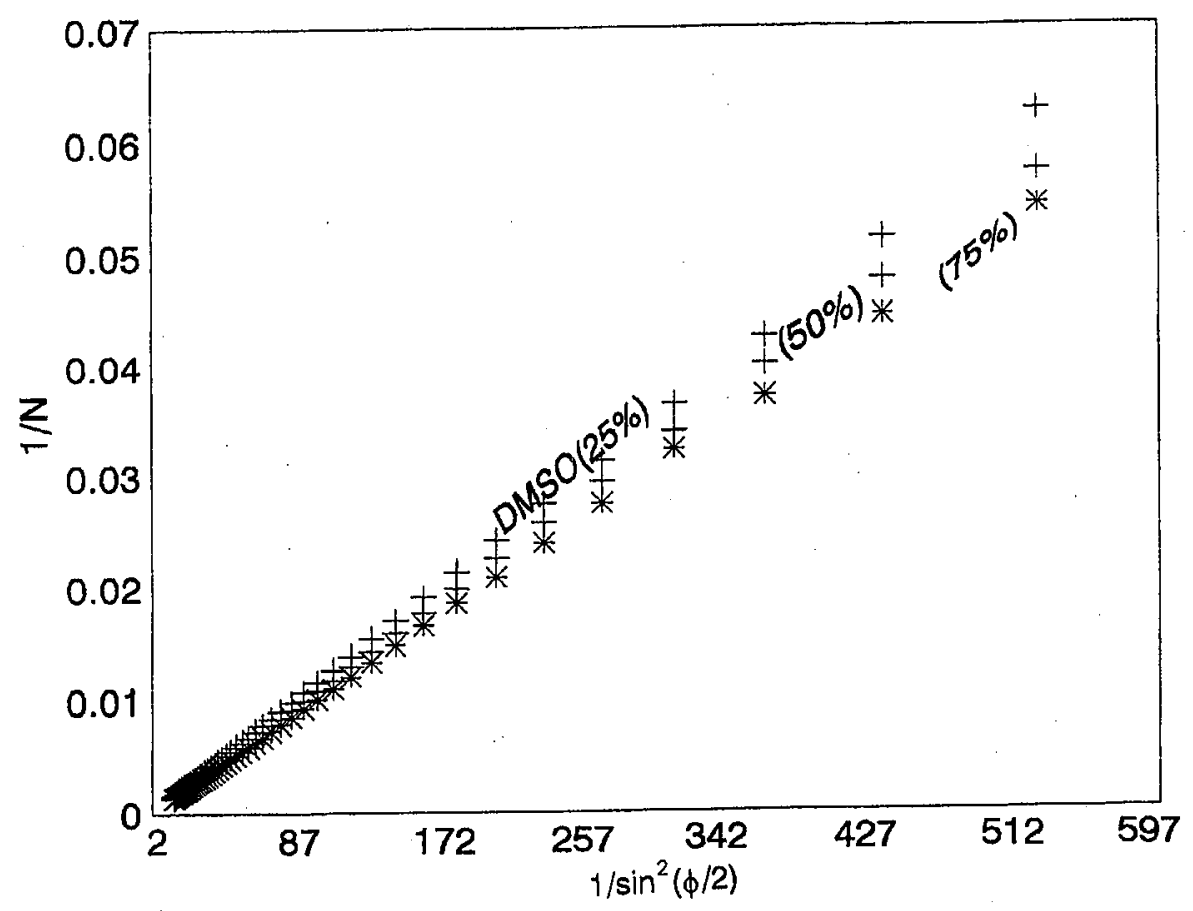

Fig. 9. Determination of the refractive index of different percentages $P 25 \%, 50 \%$, and $75 \%$. 


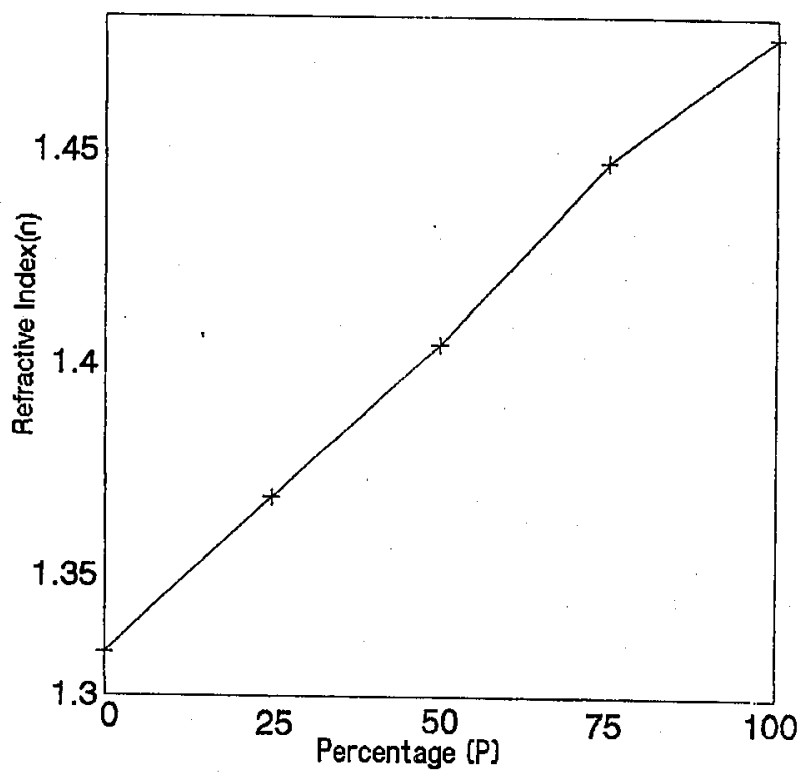

Fig. 10. The relation between percentage $P 0 \%, 25 \%, 50 \%, 75 \%$, and $100 \%$ and refractive index $n$.

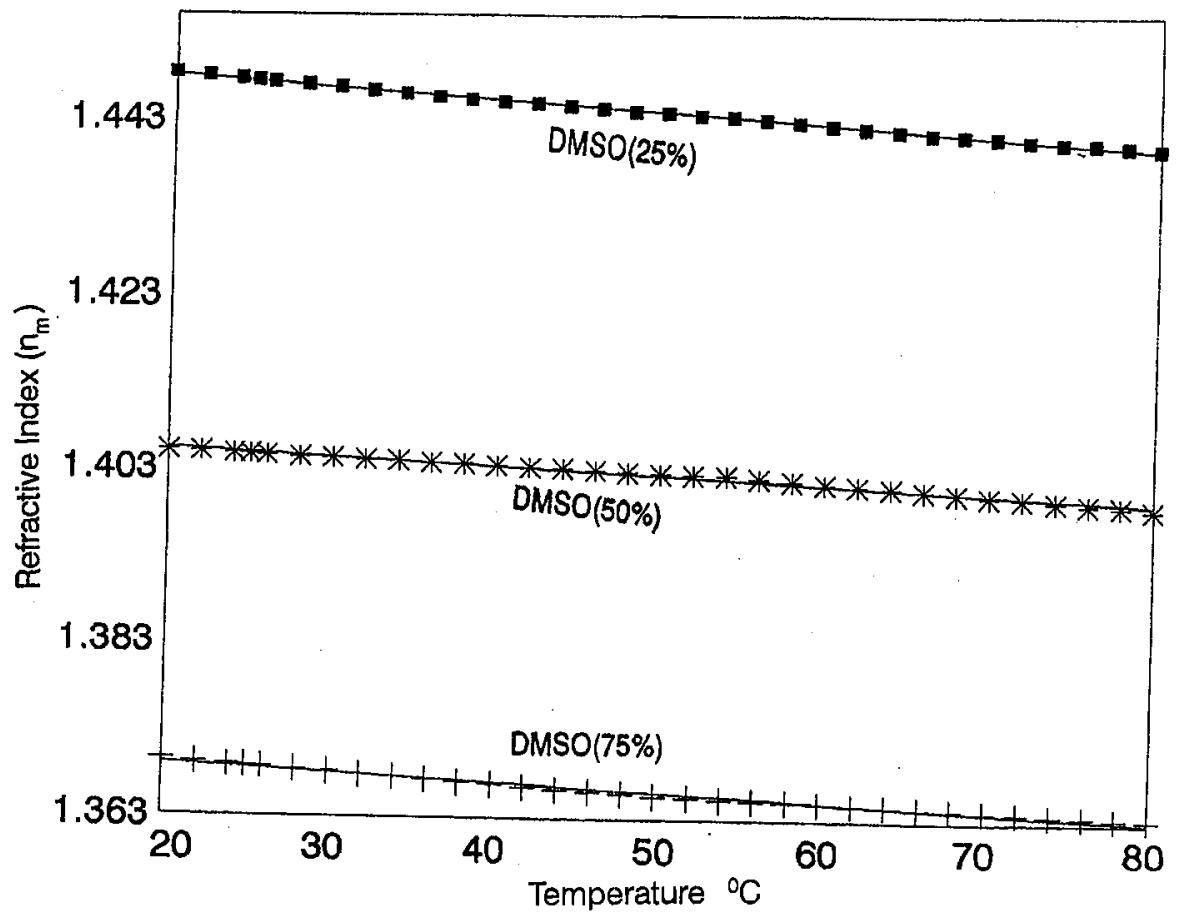

Fig. 11. Temperature behavior of refractive index of the three different percentages $25 \%, 50 \%$, and $75 \%$ DMSO. 


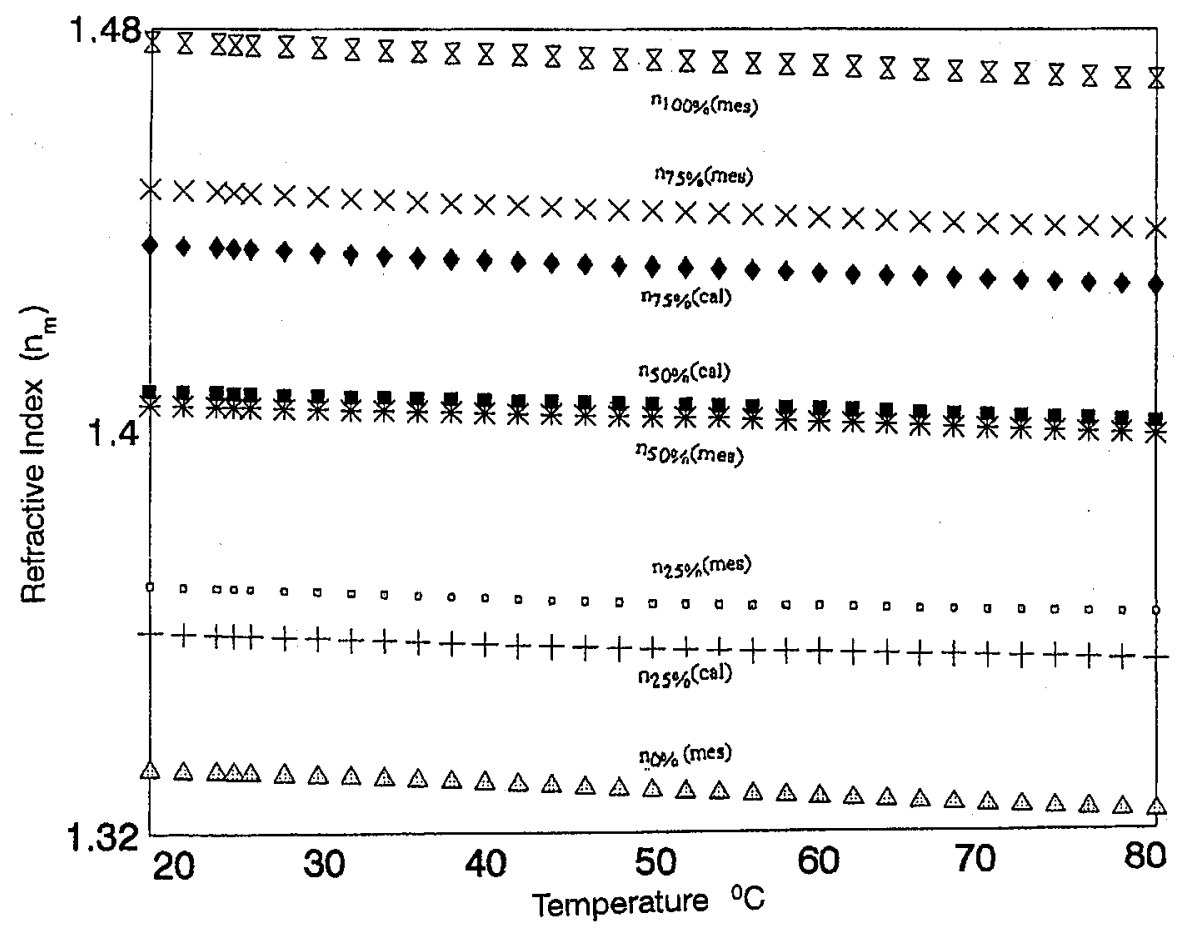

Fig. 12. Comparisons between measured and calculated values of refractive index versus temperature for different percentages of DMSO.

we had observed a nonzero $\mathrm{d} n / \mathrm{d} t$ for both water and DMSO we cannot expect a zero $\mathrm{d} n / \mathrm{d} t$ for any combination of water and dimethylsulfoxide. The results of this experimental work are shown in Fig. 10 for bulk refractive index $n_{\mathrm{m}}$ and Fig. 11 for $\mathrm{d} n / \mathrm{d} t$ 's of the mixed solvents. These results show also that both the bulk index and $\mathrm{d} n / \mathrm{d} t$ for solvent mixtures are inherited from the parent solvents in proportion to their percentages in the mixture. Therefore, we can expect that the effect of mixing two or more liquids can be simply accounted for, by adding proportionally their bulk indices and, $\mathrm{d} n / \mathrm{d} t$ 's.

We have assumed an empirical relation between the refractive index of a mixture $n_{\mathrm{m}}$, solvent percentage $P$ and temperature $t$ as follows:

$$
n_{\mathrm{m}}=a P^{b} t^{c}
$$

with

$$
0 \%<P<100 \%, \quad 20^{\circ} \mathrm{C} \leq t \leq 80^{\circ} \mathrm{C},
$$

and $a, b$ and $c$ are empirical constants:

$$
a=1.44133, b=5.0107 \times 10^{-2} \text {, and } c=3.3962 \times 10^{-3} \text {. }
$$

By using this formula we have calculated the refractive index $\left(n_{\mathrm{m}}\right)_{\text {cal }}$ at different temperatures for each percentage and compared it with the measured indices as shown in Fig. 12. 


\section{Conclusion}

By using MZI, we have measured the absolute refractive indices for two solvents, which belong to the most commonly used laser dye solvents. Also, we have measured the temperature behavior of these refractive indices for both pure water and DMSO in the temperature range from 20 to $80^{\circ} \mathrm{C}$ with an accuracy better than $5.8 \times 10^{-4}$. The quantum efficiency of dyes dissolved in water is improved by adding the organic additive [1]. The effect of these additives on $\mathrm{d} n / \mathrm{d} t$ and hence thermo-optical properties is reported here and it is found that an additive superimposes an additional $\mathrm{d} n / \mathrm{d} t$ on that of water. For liquid mixtures the resultant $\mathrm{d} n / \mathrm{d} t$ is simply the proportional average of the parents $\mathrm{d} n / \mathrm{d} t$.

\section{References}

[1] Dye Lasers, Ed. F.P. Schäfer, 3rd ed., Springer-Verlag, Berlin 1990.

[2] High-Power Dye Laser, Ed. F.G. Duarte, Springer-Verlag, Berlin 1991.

[3] W. Demtröder, Laser Spectroscopy, 2nd ed., Springer-Verlag, Berlin 1996.

[4] R. Ghazy, B. El-Baradie, in: 2nd Int. Conf. on Lasers and Applications, Cairo 1996, p. 128.

[5] M. Khashan, A. Nassif, J. Mod. Opt. 36, 785 (1989).

[6] N. Subrahmanyam, B. Lal, A Text Book of Optics, 13th ed., S. Chand and Company Ltd., New Delhi 1983.

[7] L. Onsager, J. Am. Chem. Soc. 58, 1486 (1936).

[8] M. Born, E. Wolf, Principles of Optics, 6th ed., Pergamon Press, Oxford 1993.

[9] B. Yavorsky, A. Detlaf, Handbook of Physics, Mir Publishers, Moscow 1975.

[10] L.A. Lee, R.A. Robb, IEEE QE 16, 777 (1980).

[11] M.E. Lusty, M.H. Dunn, Appl. Phys. B 44, 193 (1987).

[12] B. Richerzhagen, Appl. Opt. 35, 1650 (1996).

[13] CRC Handbook of Chemistry and Physics, Ed. R.C. Weast, 57th ed., CRC Press Inc., Boca Raton (Fl) 1976.

[14] U. Brackmann, Lambdachrome Laser Dyes, Lambda Physik GmbH, Göttingen 1986. 